\title{
An Optical Fiber Liquid Level Sensor Based on Side Coupling Induction Technology
}

\author{
Yanjun Zhang $\mathbb{D}^{1,2}$ Yingzi Zhang $\mathbb{D}^{1,2}$ Yulong Hou, ${ }^{1,2}$ Liang Zhang $\mathbb{D}^{1,2}$ Yanjun $H u\left(\mathbb{D},{ }^{1,2}\right.$ \\ Xiaolong Gao $\mathbb{D}^{1,2}$ Huixin Zhang, ${ }^{1,2}$ and Wenyi Liu $\mathbb{D}^{1,2}$ \\ ${ }^{1}$ Key Laboratory of Instrumentation Science \& Dynamic Measurement, Ministry of Education, North University of China, \\ Taiyuan 030051, China \\ ${ }^{2}$ Science and Technology on Electronic Test \& Measurement Laboratory, North University of China, Taiyuan 030051, China
}

Correspondence should be addressed to Yingzi Zhang; zhangyingzinuc@163.com

Received 18 August 2017; Accepted 3 December 2017; Published 8 January 2018

Academic Editor: Stefania Campopiano

Copyright ( 2018 Yanjun Zhang et al. This is an open access article distributed under the Creative Commons Attribution License, which permits unrestricted use, distribution, and reproduction in any medium, provided the original work is properly cited.

An optical fiber liquid level sensor based on two twisted polymer optical fibers twining around a racetrack column is demonstrated in this study. The side-coupling power of the passive fiber is modulated by the refractive index (RI) of the environment medium and decreases while the liquid level increases. The variation patterns of the side-coupling power in the bent section and the straight section form a step attenuation, which can improve the measurement range with a superior sensitivity and distinguish liquids with different RIs. Furthermore, the cost-effective sensor also shows good reversibility and low temperature-dependent properties.

\section{Introduction}

Liquid level sensing is of great significance in many fields such as in chemical industry, early warning of flood, petroleum storage, and public water supplies. In harsh (conductive or explosive) environment, safety considerations limit the applications of traditional sensors based on mechanical or electrical techniques. Considering these restrictions, optical fiber sensing has gained a lot of attention due to its strong immunity to electromagnetic interference, remote sensing, and low cost $[1,2]$.

Nowadays, optical fiber liquid level sensors in the reported literature are usually categorized into wavelengthmodulated measurement and intensity-modulated measurement. Most wavelength-modulated sensors are based on structures of fiber Bragg gratings (FBGs) [3], long-period fiber gratings (LPFGs) [4], Mach-Zehnder interferometers [5], and so on. Although the sensitivity of these sensors is high, the fabrication process is costly and complicated in general and the measurement scale is limited to dozens of millimeters. Conversely, the intensity-modulated sensors using POFs are cost effective [6], which introduces a continuously or periodically varied loss with the liquid level changing. The structure is simple and the measurement range is large enough. For example, the operational range of the multipoint liquid level sensor could exceed $2 \mathrm{~m}$, which is achieved by engraving grooves on a vertical POF $[7,8]$, bending and polishing POFs [9] or aligning a group of POF segments coaxially [10], and so on. The maximum range among them is $2.8 \mathrm{~m}$ but the resolution is $350 \mathrm{~mm}$. A continuous liquid level sensor with two twisted POFs twining around a cylindershaped column is proposed, and it has a measurement range of 350 millimeters [11]. However, the sensor is difficult to differentiate liquids with different refractive indexes (RIs).

In this paper, we propose and demonstrate a costeffective liquid level sensor based on two twisted POFs twining around a racetrack column, which expands the measurement range with a satisfactory sensitivity and can recognize the liquids with different RIs. The variations of output power is adjusted by the liquid level. The twisted macrobend coupling structure (TMBCS) improves the signal-to-noise ratio (SNR) substantially, overcoming the drawback of intensity-based sensors $[12,13]$. The fabrication process of the proposed sensor is simple and cheap. The measurement range is flexible. Meanwhile, it does not require a temperature compensation. 


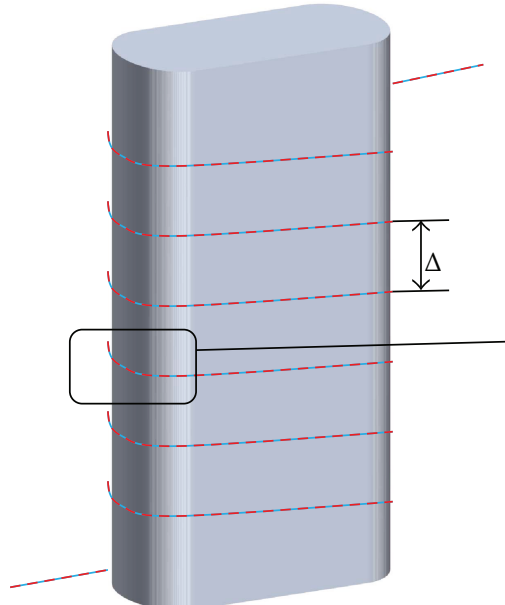

(a)

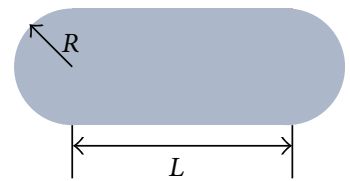

(b)

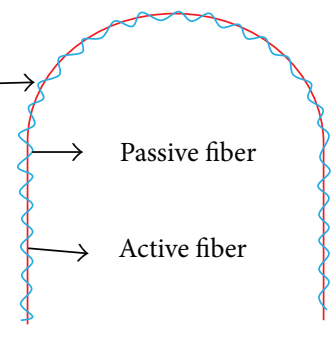

(c)

FIgURE 1: (a) The schematic diagram of the proposed liquid level sensor. (b) The planform of the racetrack column. (c) The TMBCS of two POFs.

\section{Operating Principle}

The schematic diagram of the proposed sensor is shown in Figure 1(a). To achieving the liquid level measurement, two twisted POFs twines around a racetrack column. $\Delta$ is the screw pitch. The planform of the racetrack column is depicted in Figure 1(b), where $R$ is the curvature radius of the bent section and $L$ is the length of the straight section. When light propagates in the bent section, a portion of light rays is refracted at some points of the core-environment interface and is called macrobend losses [13]. As the macrobend losses are modulated by $R$ and the RI of the environment medium, they will increase while the environment medium changes from air to liquid, and the curvature radius is a fixed value. Compared with the cylinder-shaped column, the racetrack one introduces the straight section that makes the macrobend losses change sharply in the bent section and slowly in the straight section with the liquid level rising. The alternating variations slow down the attenuation of the light power in POF and enlarge the measurement range. Also, the proposed sensor can distinguish liquids with different RIs according to the sensitivity in the bent and straight section.

Although the macrobend losses can be improved by decreasing $R$, their power is still very weak compared with the power of the light source, and the sensing signal is easy to be drowned in the light source fluctuation. Thus, a TMBCS is applied here. As can be seen in Figure 1(c), the active fiber, connected to the light source, is tightly twisted with the passive fiber and then bent to a semicircle. A side-coupling region is established between two macrobend POFs, and a portion of the macrobend losses from the active fiber transfers to the passive fiber in the region, which is called the side-coupling power. Since the energy of the light source is mainly concentrated in the core of the fiber, the sidecoupling ratio is very small, and the fluctuations of the light source are well restricted into the active fiber. It has been proved in [12] that the dark-field characteristics of the side-coupling power make the influence of the light source fluctuation negligible when a stable LED light source is used, and the visible light fluctuations become the major part of the background noises. Therefore, the optical isolation is needed, and a better SNR can be attained by detecting the side-coupling power. In addition, the side-coupling power is more easily modulated by the RI of the environment medium. For a racetrack column with fixed geometric parameters, the output power at the end of the passive fiber decreases with a step trend regularly while the liquid level increases continuously. The proposed sensor works on this principle that is called the side-coupling induction technology. By measuring the side-coupling power, both the SNR of the proposed sensor and the sensitivity to the ambient environment are improved.

\section{Experiment}

The experimental setup of the liquid level sensor is shown in Figure 2(a). The setup consists of a $660 \mathrm{~nm}$ fibercoupled LED (M660F1, Thorlabs) light source, two optical power meters (PM100USB, Thorlabs), two twisted POFs (Mitsubishi, SK40) twining around a racetrack column with a helical structure, and a testing container. To achieve the visible light shielding, the insensitive regions of POFs and the free end are put into the black jacket. The arrangement of measurement points is shown in Figure 2(b), where ten measurement points are distributed on average between two adjacent turns on the same side. Thus, the height difference of the two adjacent measurement points is $\Delta / 10$. During the experiment, the light source power was set at $30 \mathrm{~mW}$ and the resolution of the power meter was $0.1 \mathrm{nW}$. The liquid level changed from the lowest to the highest gradually and the corresponding output power of the passive fiber was detected and recorded when the liquid reached the measurement points. Water is selected as the test liquid, and all the experiments are conducted in the dark room. 


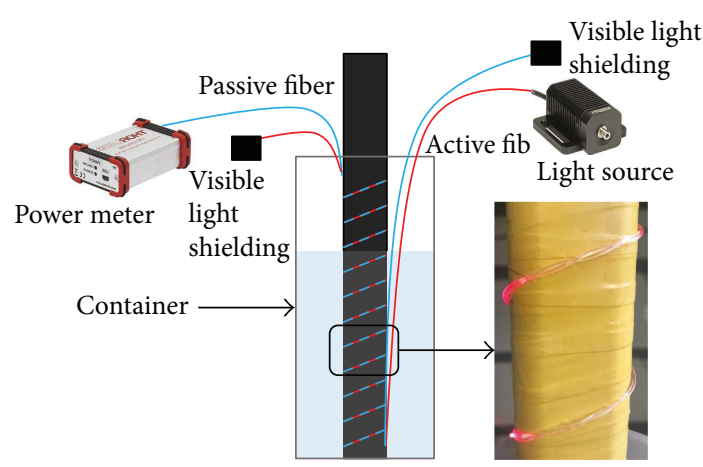

(a)

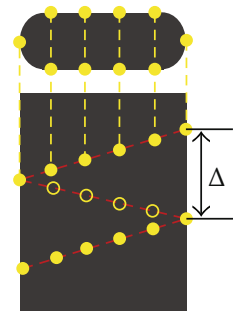

(b)

Figure 2: (a) Experimental setup of the proposed liquid level sensor. (b) The arrangement of measurement points in the bent and straight section of the POF.

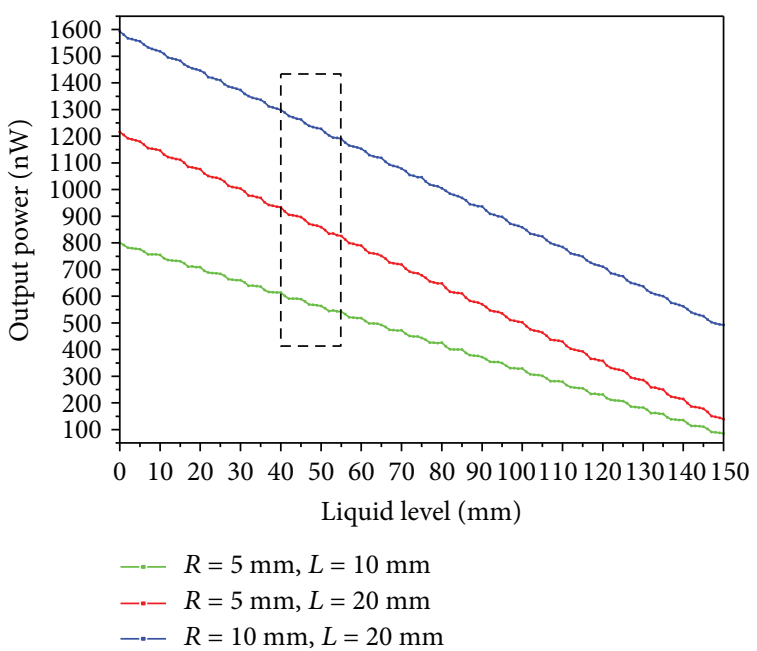

(a)

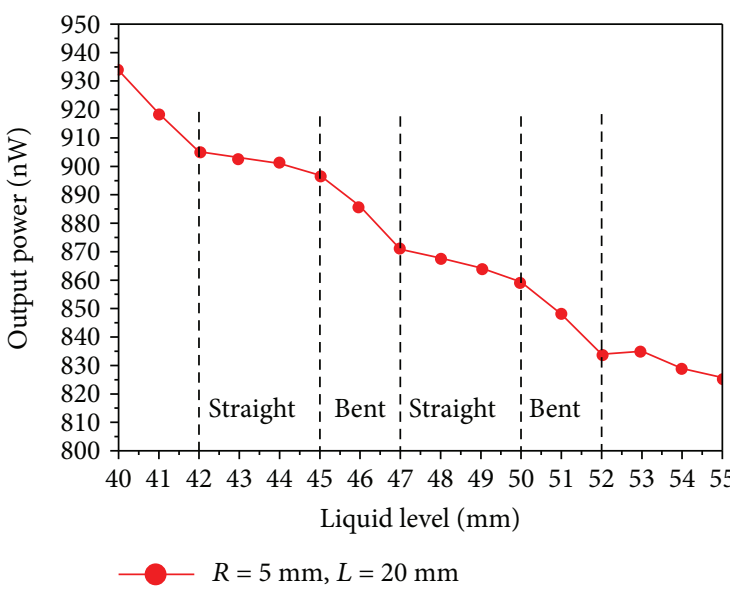

(c)

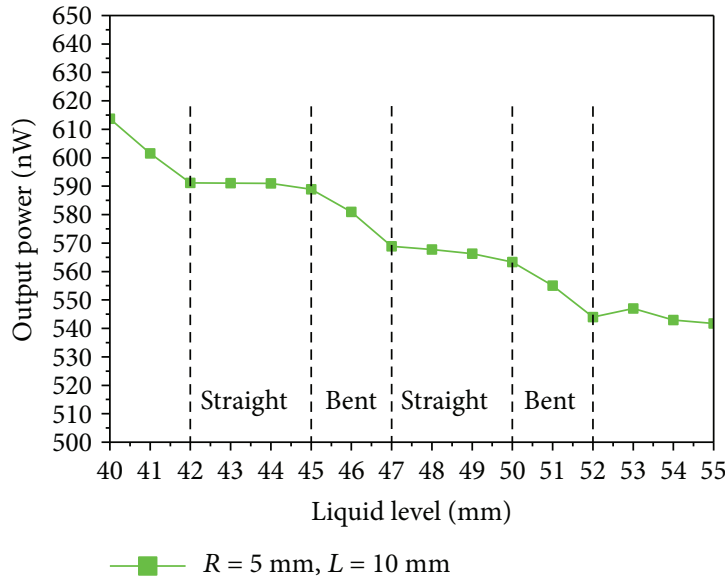

(b)

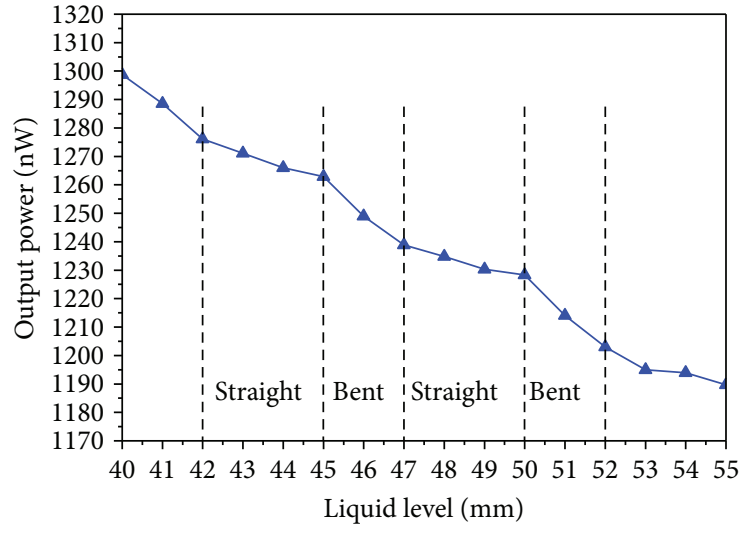

$R=10 \mathrm{~mm}, L=20 \mathrm{~mm}$

(d)

FIgURe 3: The liquid level response of the proposed sensor with different geometric parameters of the racetrack column. (a) Comparison of the liquid level response for three sizes; (b) $R=5 \mathrm{~mm}$ and $L=10 \mathrm{~mm}$; (c) $R=5 \mathrm{~mm}$ and $L=20 \mathrm{~mm}$; (d) $R=10 \mathrm{~mm}$ and $L=20 \mathrm{~mm}$. $\Delta=10 \mathrm{~mm}, n_{2}=1.334$, and $u=0.03 \mathrm{nW} / \mathrm{mm}$.

\section{Results and Discussion}

The performance of the proposed sensor with different geometric parameters is investigated and the results are depicted in Figure 3. The screw pitch of the racetrack helical structure is $10 \mathrm{~mm}$ and the variation range of the liquid level is $150 \mathrm{~mm}$. With the liquid level increasing, the output power of the power meter decreases fast in the bent section and slow 


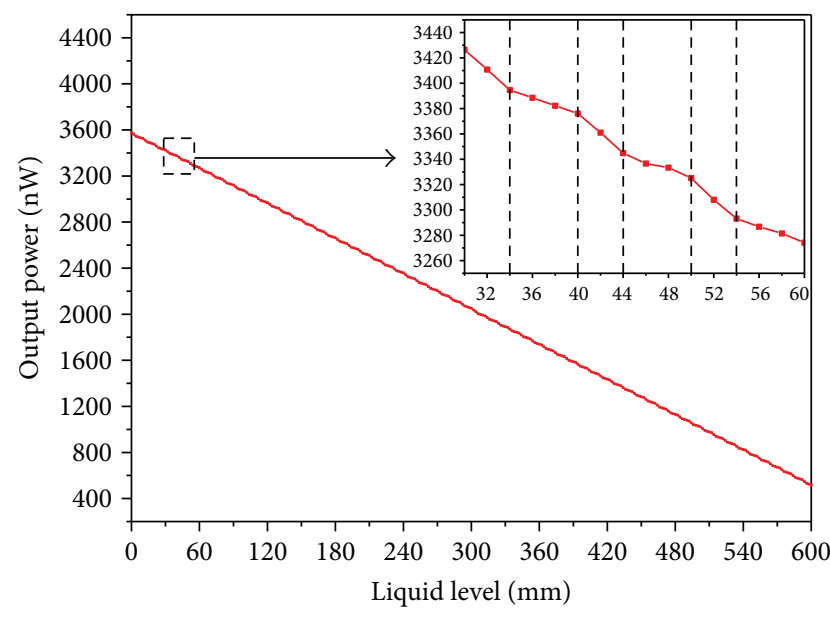

$-\cdot-\Delta=20 \mathrm{~mm}$

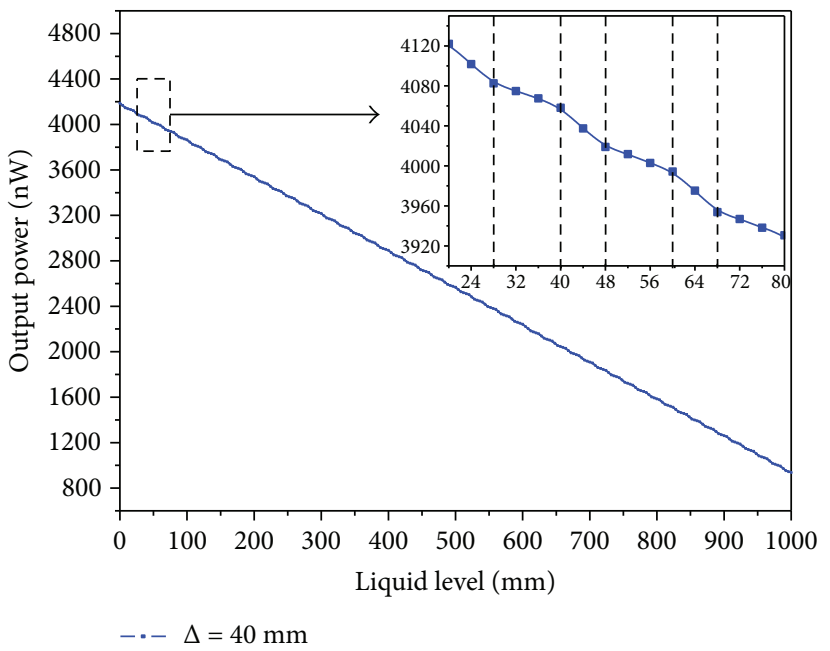

(b)

FIGURE 4: The measurement range of the proposed sensor, where the screw pitch $\Delta$ are (a) $20 \mathrm{~mm}$ and (b) $40 \mathrm{~mm} . R=10 \mathrm{~mm}, L=20 \mathrm{~mm}$, $n_{2}=1.334$, and $u=0.03 \mathrm{nW} / \mathrm{mm}$.

in the straight section, forming a step attenuation. In Figure 3(b) and Figure 3(c), when $R$ is $5 \mathrm{~mm}$ and $L$ varies from 10 to $20 \mathrm{~mm}$, the sensitivity of the bent section and the straight section changes from 10.18 and 0.92 to 12.97 and $3.01 \mathrm{nW} / \mathrm{mm}$, respectively. It can be deduced that the increase of $L$, which enlarges the coupling length of the twisted POFs between two neighboring measurement points, will improve the sensitivity. And comparing Figure 3(c) to Figure 3(d), the macrobend losses of the bent section decrease while $R$ increases from 5 to $10 \mathrm{~mm}$. As a result, the sensitivity of the bent section declines from 12.97 to $11.89 \mathrm{nW} / \mathrm{mm}$ and the measurement range can be expanded. In the meantime, the increase of $R$ will make the coupling length of two adjacent measurement points extend slightly, increasing the sensitivity of the straight section from 3.01 to $3.96 \mathrm{nW} / \mathrm{mm}$. To sum up, the variation of $R$ will affect the measurement range and the sensitivity can be improved by decreasing $R$ or enlarging the coupling length between two adjacent measurement points.

The measurement range of the proposed sensor with different screw pitch is illustrated in Figure 4. In Figure 4(a), when $\Delta$ changes from 10 to $20 \mathrm{~mm}$, the measurement scale expands from 150 to $600 \mathrm{~mm}$. But the sensitivity of the bent section and the straight section decreases from 11.89 and 3.96 to 7.94 and $3.02 \mathrm{nW} / \mathrm{mm}$. Figure 4(b) shows that the measurement range could reach $1000 \mathrm{~mm}$ when $\Delta$ is $40 \mathrm{~mm}$. Correspondingly, the sensitivity declines to $5.01 \mathrm{nW} / \mathrm{mm}$ in the bent section and $1.99 \mathrm{nW} / \mathrm{mm}$ in the straight section. Under a constant liquid level, the increase of $\Delta$ will reduce the number of turns submerged in the liquid, which decreases the macrobend losses and expands the measurement range. In the meantime, the sensitivity declines because of the diminution of the coupling length.

As can be seen in Figure 5, water, 9.1\% $\mathrm{NaCl}$ solution, and $16.7 \% \mathrm{NaCl}$ solution are used as the specimens to examine the proposed liquid level sensor. For the liquid with indices of $1.334,1.348$, and 1.360 , the results show that

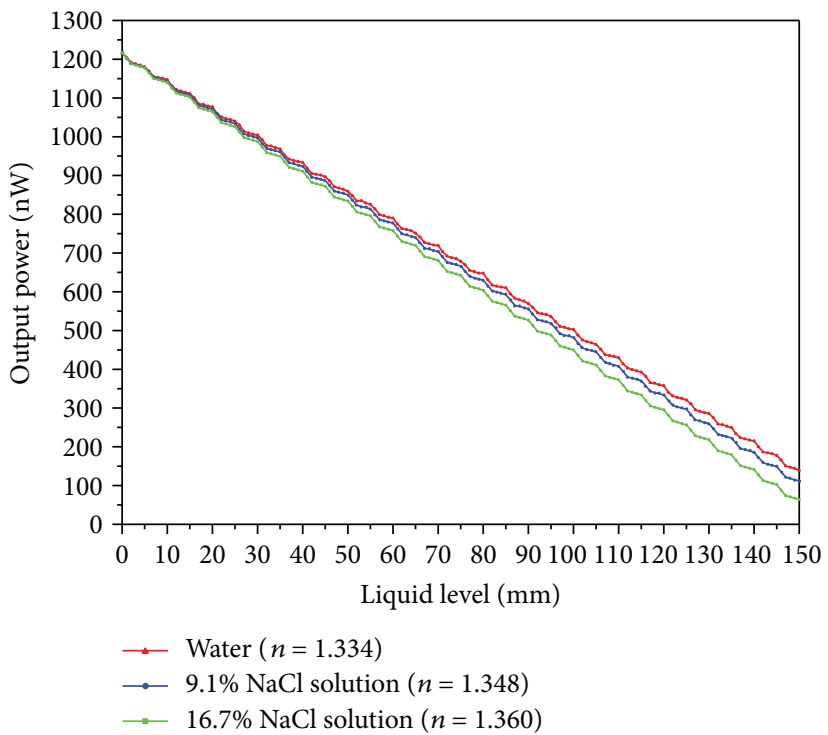

FIgURE 5: The liquid leveling sensing for liquids with different refractive indices. $R=5 \mathrm{~mm}, L=20 \mathrm{~mm}, \Delta=10 \mathrm{~mm}$, and $u=$ $0.03 \mathrm{nW} / \mathrm{mm}$.

the sensitivities of the bent section are 12.97, 13.42, and $14.12 \mathrm{nW} / \mathrm{mm}$ and those of the straight section are 3.01 , 3.22 , and $3.58 \mathrm{nW} / \mathrm{mm}$. As expected, the sensitivity of the proposed sensor is related to the RI of the liquid and a higher RI shows a higher sensitivity. This is because the increase of the RI of the test liquid leads to more macrobend losses at every turn.

The liquid level responses to the ascending order and descending order are shown in Figure 6. It can be seen that the sensitivities of the bent and straight section are 12.97 and $2.99 \mathrm{nW} / \mathrm{mm}$ with the descending order, which are almost the same as those of the ascending order. To improve the consistency of the measuring results and the 


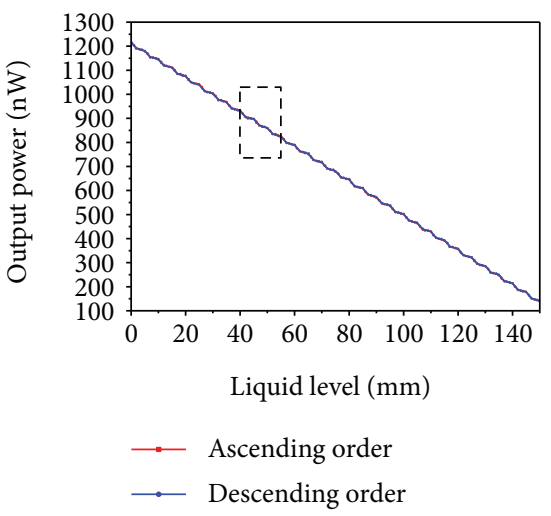

(a)

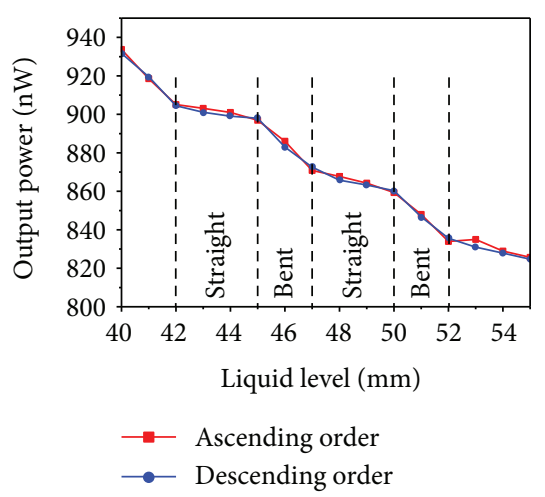

(b)

Figure 6: The reversibility of the proposed sensor. $R=5 \mathrm{~mm}, L=20 \mathrm{~mm}, \Delta=10 \mathrm{~mm}, n_{2}=1.334$, and $u=0.03 \mathrm{nW} / \mathrm{mm}$.

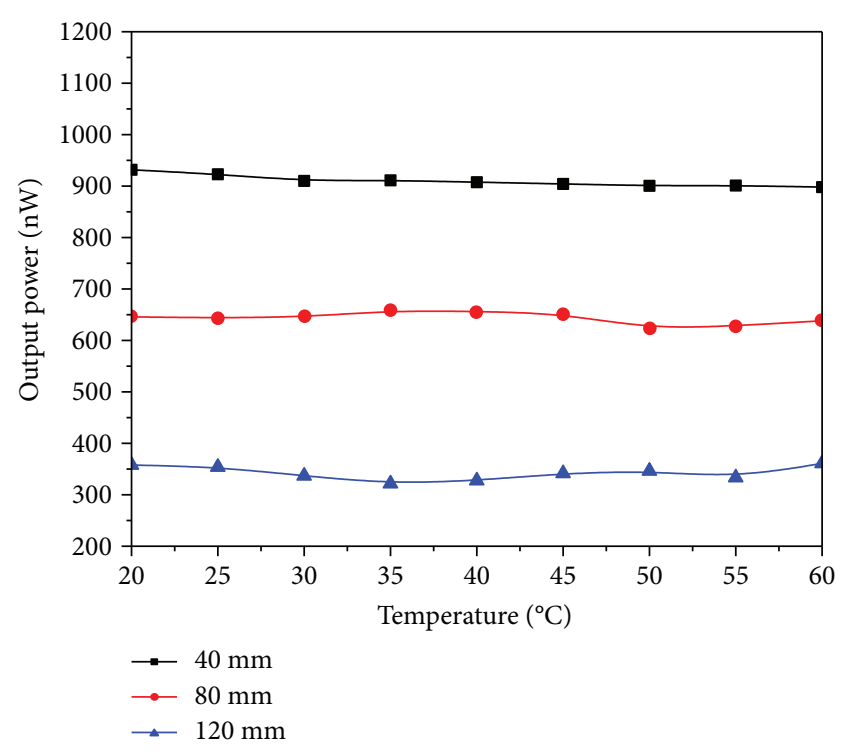

Figure 7: The temperature dependence of the sensor under different liquid levels. $R=5 \mathrm{~mm}, L=20 \mathrm{~mm}, \Delta=10 \mathrm{~mm}$, and $n_{2}=1.334$.

robustness of the sensor, the two POFs are twisted tightly, making the relative position of the two fibers stable and the center-to-center spacing small enough. Thus, little liquid can exist in the gap between two POFs while the liquid level decreases. Therefore, the reversibility of the sensor is satisfying.

Finally, the container is placed on a heater to investigate the response of temperature. As depicted in Figure 7, the measurements are performed under different liquid levels which are 40,80 , and $120 \mathrm{~mm}$, respectively. The temperature increases from 20 to $60^{\circ} \mathrm{C}$ with an interval of $5^{\circ} \mathrm{C}$. The output power is fluctuant irregularly in a small range, which has little influence on the liquid level sensing of the proposed sensor. It can be deduced that, for two twisted POFs with a certain racetrack helical structure, the side-coupling power is temperature-independent and mainly modulated by the RI of the environment medium.

\section{Conclusions}

In conclusion, an optical liquid level sensor based on SCIT has been investigated. The sensor is constructed by just twining two twisted POFs around the racetrack column, which is simple and cost effective. The liquid level sensing is achieved by measuring the side coupling power that changes with the $\mathrm{RI}$ of the environment medium. With the liquid level rising, the side coupling power shows a step attenuation. Thus, the proposed sensor could differentiate the liquids and expand the measurement range. The sensitivity and the measurement range are determined by the parameters of the racetrack helical structure. Moreover, the sensor has merits of good reversibility and temperature independence. As mentioned previously, the sensitivity of the wavelength-based sensor is high, but the measurement range is too narrow. When the measurement range is large enough, the resolution is unsatisfactory. Therefore, the proposed sensor is an acceptable choice considering the sensing range and sensitivity. Our future research will focus on the improvement of the sensitivity or the measurement scale.

\section{Conflicts of Interest}

The authors declare that there is no conflict of interest regarding the publication of this article.

\section{Acknowledgments}

This work was sponsored by the Fund for Shanxi "1331 Project" Key Subject Construction. This work was supported by the Natural Science Foundation of Shanxi Province, China (201701D121065).

\section{References}

[1] B.-M. M. Barerem-Melgueba Mao, Y. W. Yizhen Wei, and B. Z. Bin Zhou, "Comparative study of fiber optic liquid level sensors based on long-period fiber gratings with different doping concentrations," Chinese Optics Letters, vol. 10, no. 5, pp. 050501-050504, 2012. 
[2] J. Zubia, U. Irusta, J. Arrue, and A. Aguirre, "Design and characterization of a plastic optical fiber active coupler," IEEE Photonics Technology Letters, vol. 10, no. 11, pp. 15781580, 1998.

[3] C. Li, T. Ning, C. Zhang et al., "Liquid level measurement based on a no-core fiber with temperature compensation using a fiber Bragg grating," Sensors and Actuators A: Physical, vol. 245, pp. 49-53, 2016.

[4] K. Ren, L. Ren, J. Liang et al., "Online fabrication scheme of helical long-period fiber grating for liquid-level sensing," Applied Optics, vol. 55, no. 34, pp. 9675-9679, 2016.

[5] C. Li, T. Ning, C. Zhang, X. Wen, J. Li, and C. Zhang, "Liquid level and temperature sensor based on an asymmetrical fiber Mach-Zehnder interferometer combined with a fiber Bragg grating," Optics Communications, vol. 372, pp. 196-200, 2016.

[6] J. Zubia and J. Arrue, "Plastic optical fibers: an introduction to their technological processes and applications," Optical Fiber Technology, vol. 7, no. 2, pp. 101-140, 2001.

[7] P. Antunes, J. Dias, T. Paixão, E. Mesquita, H. Varum, and P. André, "Liquid level gauge based in plastic optical fiber," Measurement, vol. 66, pp. 238-243, 2015.

[8] E. Mesquita, T. Paixão, P. Antunes et al., "Groundwater level monitoring using a plastic optical fiber," Sensors and Actuators A: Physical, vol. 240, pp. 138-144, 2016.

[9] M. Lomer, J. Arrue, C. Jauregui, P. Aiestaran, J. Zubia, and J. M. López-Higuera, "Lateral polishing of bends in plastic optical fibres applied to a multipoint liquid-level measurement sensor," Sensors and Actuators A: Physical, vol. 137, no. 1, pp. 68-73, 2007.

[10] X. Lin, L. Ren, Y. Xu et al., "Low-cost multipoint liquid-level sensor with plastic optical fiber," IEEE Photonics Technology Letters, vol. 26, no. 16, pp. 1613-1616, 2014.

[11] H. Zhang, L. Feng, Y. Hou et al., "Optical fiber liquid level sensor based on macro-bending coupling," Optical Fiber Technology, vol. 24, pp. 135-139, 2015.

[12] Y. L. Hou, W. Y. Liu, S. Su et al., "Polymer optical fiber twisted macro-bend coupling system for liquid level detection," Optics Express, vol. 22, no. 19, pp. 23231-23241, 2014.

[13] Y. Zhang, Y. Hou, W. Liu et al., "A cost-effective relative humidity sensor based on side coupling induction technology," Sensors, vol. 17, no. 5, p. 944, 2017. 


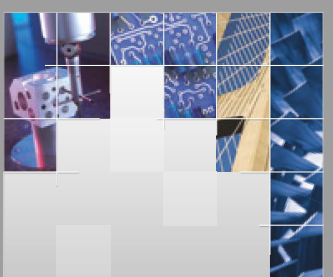

\section{Enfincering}
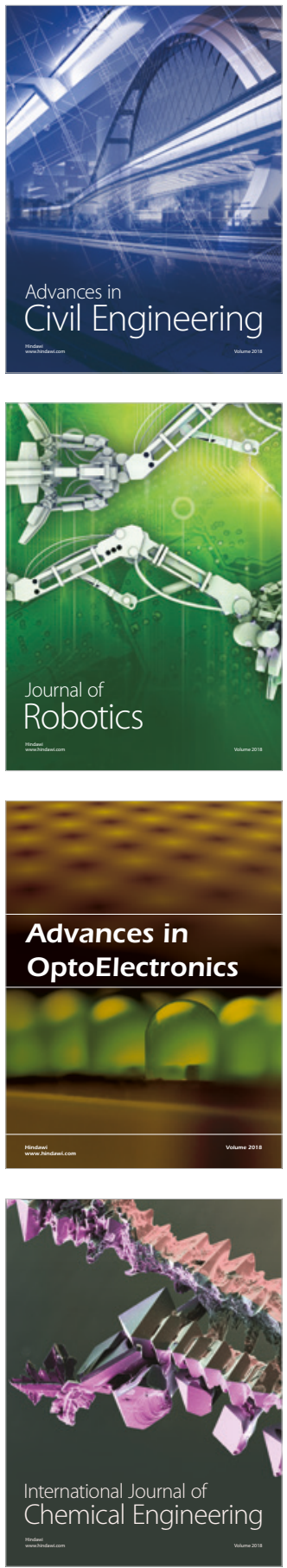

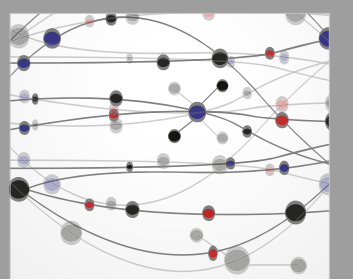

\section{Rotating \\ Machinery}

The Scientific World Journal

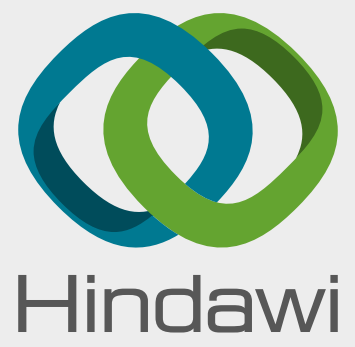

Submit your manuscripts at

www.hindawi.com
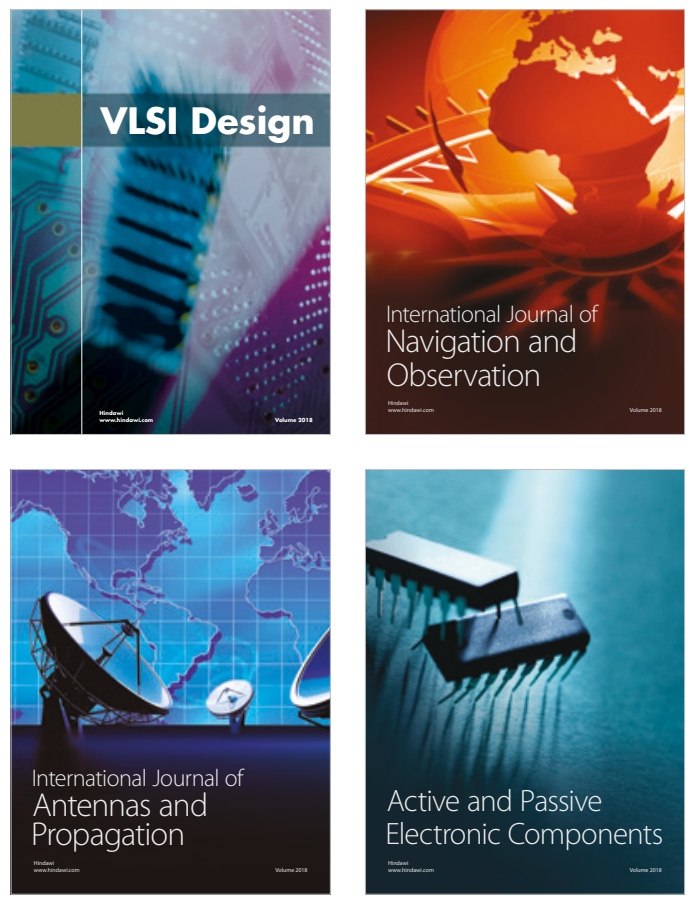
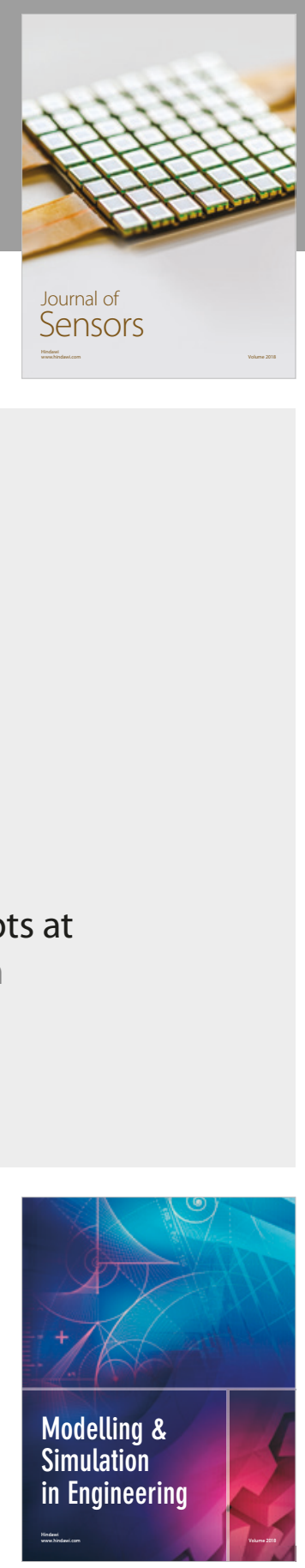

\section{Advances \\ Multimedia}
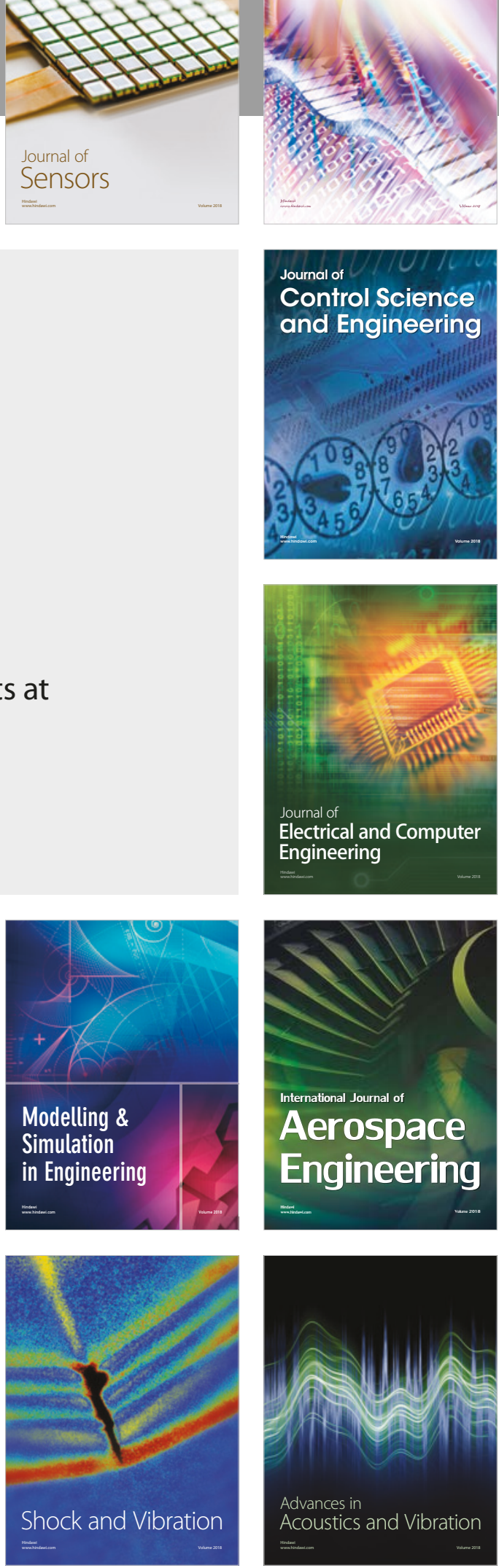